\title{
CT Perfusion in Acute Lacunar Stroke: Detection Capabilities Based on Infarct Location
}

\author{
(D).C. Benson, (D) S. Payabvash, (D) S. Mortazavi, (D). Zhang, (D) P. Salazar, (D) B. Hoffman, (D) M. Oswood, and (D) A.M. McKinney
}

\begin{abstract}
BACKGROUND AND PURPOSE: Recent studies demonstrated superiority of CTP to NCCT/CTA at detecting lacunar infarcts. This study aimed to assess CTP's capability to identify lacunae in different intracranial regions.
\end{abstract}

MATERIALS AND METHODS: Over 5.5 years, 1085 CTP examinations were retrospectively reviewed in patients with acute stroke symptoms with CTP within 12 hours and MRI within 7 days of symptom onset. Patients had infarcts $\leq 2 \mathrm{~cm}$ or no acute infarct on DWl; patients with concomitant infarcts $>2 \mathrm{~cm}$ on DWI were excluded. CTP postprocessing was automated by a delay-corrected algorithm. Three blinded reviewers were given patient NIHSS scores and symptoms; infarcts were recorded based on NCCT/CTA, CTP (CBF, CBV, MTT, and TTP), and DWI.

RESULTS: One hundred thirteen patients met inclusion criteria (53.1\% female). On DWI, lacunar infarcts were present in 37 of 113 (32.7\%), and absent in 76 of 113 (67.3\%). On CTP, lacunar infarcts typically appeared as abnormalities larger than infarct size on DWI. Interobserver $\kappa$ for CTP ranged from 0.38 (CBF) $(P<.0001)$ to 0.66 (TTP) $(P<.0001)$; interobserver $\kappa$ for DWI was $0.88(P<0.0001)$. In all intracranial regions, sensitivity of CTP ranged from 18.9\% (CBV) to 48.7\% (TTP); specificity ranged from 97.4\% (CBF and TTP) to 98.7\% (CBV and MTT). CTP's sensitivity was highest in the subcortical white matter with or without cortical involvement $(21.7 \%-65.2 \%)$ followed by periventricular white matter (12.5\%-37.5\%); sensitivity in the thalami or basal ganglia was $0 \%$.

CONCLUSIONS: CTP has low sensitivity and high specificity in identifying lacunar infarcts. Sensitivity is highest in the subcortical white matter with or without cortical involvement, but limited in the basal ganglia and thalami.

ABBREVIATIONS: CSWM = subcortical white matter with or without involvement of the cortex; PVWM = periventricular white matter

$\mathbf{L}^{2}$ acunar infarcts are ischemic insults that predominantly result from the occlusion of single perforating arteries. ${ }^{1}$ Typically, lacunae occur within the thalami, basal ganglia, brain stem, corona radiata, or internal capsule. ${ }^{2-4}$ Although lacunar infarcts predominantly occur secondary to small vessel disease, other etiologies such as cardiac emboli and vasculitis also have been described. ${ }^{5}$ Lacunar strokes are a common cause of morbidity and account for up to $25 \%$ of all ischemic strokes. ${ }^{6,7}$ Classically, symp-

Received April 5, 2016; accepted after revision June 27

From the Department of Radiology (J.C.B., S.P., S.M., A.M.M.) and Clinical and Translational Science Institute (L.Z., P.S.), University of Minnesota Medical Center, Minneapolis, Minnesota; Vital Images, a division of Toshiba Medical (B.H., M.O.), Minnetonka, Minnesota; and Department of Radiology (B.H., M.O.), Hennepin County Medical Center, Minneapolis, Minnesota.

Paper previously presented at: Annual Meeting of the American Society of Neuroradiology, May 23-26, 2016; Washington, DC.

All authors have participated sufficiently to take public responsibility for content.

Please address correspondence to John C. Benson, MD, University of Minnesota Medical Center, MMC 292, B-212 Mayo, 420 Delaware St SE, Minneapolis, MN 55455; e-mail: benso905@umn.edu

http://dx.doi.org/10.3174/ajnr.A4904 toms of lacunar infarcts include pure sensory syndrome, pure motor hemiparesis, sensorimotor stroke, ataxic hemiparesis, or dysarthria-clumsy hand syndrome. ${ }^{2,8}$ Thrombolytic therapy has been shown to be effective in the treatment of acute lacunar stroke, making the timely and accurate diagnosis of lacunar infarcts of utmost importance. ${ }^{9}$

NCCT is less sensitive than DWI in the detection of lacunar strokes, with reported sensitivities ranging from $0 \%-35 \%$ for NCCT and 75\%-95\% for DWI. ${ }^{10-13}$ Nevertheless, because CT remains more accessible than MR imaging in emergency settings, its optimization in the diagnosis of stroke continues to be vital. ${ }^{14}$ Recently, CTP has been shown to be superior to NCCT and CTA in assessing for lacunar infarcts. ${ }^{4,15}$ However, neither of the recent studies directly compared CTP with NCCT/CTA in the detection of lacunae: Rudilosso et $\mathrm{al}^{15}$ assessed the use of CTP in patients with clinical lacunar syndrome, and Das et $\mathrm{al}^{4}$ investigated the use of CTP as part of a multimodal approach in the setting of lacunar infarction, noting only that $61 \%$ of patients had an abnormality on CTP concordant with infarction on DWI. Furthermore, nei- 
ther study investigated CTP's capability to detect lacunae within specific regions of the brain.

The purpose of this study was to compare the diagnostic performance of CTP with NCCT/CTA in the identification of acute lacunar infarcts and evaluate the diagnostic capabilities of CTP in the detection of lacunar strokes within different areas of the brain.

\section{MATERIALS AND METHODS \\ Patient Selection}

This study was completed at a level 1 trauma center and primary stroke center. Institutional review board approval was obtained for this study. A retrospective review of all patients who presented with symptoms of acute stroke between January 2006 and July 2011 was performed; 1085 patients with CTP examinations were identified. Patients were included if they underwent acute CTP imaging within 12 hours of symptom onset, as well as DWI within 7 days of presentation. One investigator manually measured the longest axial diameter of foci of restricted diffusion on DWI, if present. Patients were included if they had either 1) negative DWI or 2) one or more infarcts $<20 \mathrm{~mm}$ in maximum diameter on DWI. The size of $20 \mathrm{~mm}$ as the uppermost criterion for lacunar infarcts was chosen based on prior studies implicating this as a clinically applicable measurement. ${ }^{4,16}$ Exclusion criteria were: 1 ) infarct $>20 \mathrm{~mm}$ in diameter, 2) lacunar stroke with coexisting large (>20 mm maximum diameter) infarct, 3) nonoptimal quality of either CTP or DWI (eg, technical problems such as motion), and 4) administration of intravenous tPA between the time of CTP and DWI acquisitions.

\section{DWI and CTP Acquisition and Postprocessing}

MR imaging scans were performed on 2 different scanners (Ingenia 1.5T and Ingenia 3T MR scanners; Philips Medical Systems, Best, the Netherlands). On the $1.5 \mathrm{~T}$ scanner, the sequence parameters were: $\mathrm{TR}=3526, \mathrm{TE}=92$, flip angle $=90^{\circ}$, EPI factor $=45$, 2.4 acceleration (SENSE) factor, 3 directions of measurement, 16 cm FOV, $131 \times 131$ matrix, 5-mm section thickness. On the $3 \mathrm{~T}$ scanner, the sequence parameters were: $\mathrm{TR}=3832$, $\mathrm{TE}=84$, flip angle $=90^{\circ}$, EPI factor $=51,2.4$ acceleration (SENSE) factor, 3 directions of measurement, $14 \mathrm{~cm}$ FOV, $152 \times 121$ matrix, 4-mm section thickness. Both scanners used 16-channel head coils. Foci of diffusion were measured in the longest axial axis. DWI b-value was $b=1000$ for all studies.

Every patient was imaged as part of the same protocol used for emergent strokes at our institution: an NCCT was immediately followed by multidetector CTA; CTP images were obtained $<5$ minutes later. For NCCT, FOV was $25 \mathrm{~cm}$, with $512 \times 512$ matrix and 3-mm sections; for CTA, FOV was $22 \mathrm{~cm}$, with $512 \times 512$ matrix and $0.9-\mathrm{mm}$ sections. CTA is performed before CTP to minimize venous contamination on CTA scans; in several years' experience with this imaging protocol, the venous contamination noted on CTP images is minimal. ${ }^{17}$ CTP images were not obtained if hemorrhage was visualized on the NCCT by the daytime hours staff, on-site radiology resident, or neuroradiology fellow; patients with hemorrhage did not meet the study's inclusion criteria.

A 64-section multidetector scanner was used to perform all CT scans (Brilliance CT; Philips Medical Systems). All CTP sequences were completed by using 2 first-pass acquisitions of sequential 55-60 second dynamic injections/scans ( 1 image/s) with no delay between the acquisitions. Two acquisitions were completed because each included $40 \mathrm{~mm}$ of coverage; hence, 1 acquisition was set at the level of the basal ganglia, and the second was set at the level of the superior margin of the ventricles. According to the manufacturer, 55-60 second dynamic acquisitions were used based on several years' experience before this study of dynamic timing that minimized truncation effect. A truncation is applied to exclude the recirculation for the computation of $\mathrm{CBV}$, with only the first-pass contrast taken into account for CBV. Each acquisition yielded 4 contiguous sections $(25 \mathrm{~cm}$ FOV, $512 \times 512$ matrix, 10 -mm section thickness, $32 \times 1.25 \mathrm{~mm}$ collimation, 80 $\mathrm{kV}, 120 \mathrm{mAs}$ ), with a total of $40 \mathrm{~mm}$ of coverage. A bolus of $36-40$ $\mathrm{mL}$ of nonionic contrast (iohexol [Omnipaque $350 \mathrm{mg} / \mathrm{mL}$; GE Healthcare, Piscataway, New Jersey]) was intravenously administered for each acquisition via a power injector at a rate of $6.0 \mathrm{~mL} / \mathrm{s}$ for a total of 72-80 $\mathrm{mL}$ of intravenous contrast for each CTP scan. A saline flush was administered between the acquisition of CTA and CTP imaging, but not between the 2 CTP acquisitions. Typically, the inferior margins of each CTP acquisition were the inferior margin of the basal ganglia and the midcentrum semiovale superiorly. The margins were occasionally adjusted if a suspected infarct was identified on the NCCT by the radiology resident or neuroradiology fellow.

CTP postprocessing was completed on a Vitrea workstation (Vital Images, Minnetonka, Minnesota). CTP postprocessing was automated by a delay-corrected singular value decomposition algorithm; the workstation's postprocessing software uses a deconvolution-based delay-corrected singular value decomposition algorithm via interhemispheric comparison (side-to-side). The interhemispheric comparison performed by the postprocessing software is done by using a multistep approach: 1) the midline of the brain is automatically located (but can be manually corrected, if necessary), 2) the affected side is automatically identified by using TTP maps, and 3) MTT, TTP, CBV, and CBF in the affected side are automatically compared with the corresponding parameters in the contralateral side by using relative differences between each voxel. Arterial input functions were selected from the anterior cerebral artery, and venous functions were selected from the posterior segment of the superior sagittal sinus; automatic selection was used, which was verified before the finalization of images.

\section{Image Review and Interpretation}

Three staff neuroradiologists ( 2 with $>10$ years' experience, 1 with 1.5 years' experience), who had been blinded to the final results, independently reviewed the DWI, NCCT, CTA, and CTP images. Each reviewer was given the patient NIHSS score and symptoms at the time of presentation; the reviewers were otherwise blinded to the patient interpretative reports and the other reviewers' interpretations. The neuroradiologists assessed for the presence of stroke by using 4 different independent CTP parameters: CBF, CBV, MTT, and TTP. Presence or absence of stroke was then assessed on all imaging sequences; NCCT, CTA, and CTP images were reviewed first, and DWI was reviewed afterward to blind the review of CTA and perfusion data to the DWI results. Stroke locations were divided into the subcortical white matter 
with or without involvement of the overlying cortex (CSWM), periventricular white matter (PVWM), basal ganglia and/or thalamus, cerebellum, and brain stem. Brain stem and cerebellar infarcts $(n=9)$ were ultimately excluded because the CTP margins did not extend inferiorly enough to encompass strokes in those locations; 1 infarct in the PVWM was also excluded because the CTP images did not fully include the infarct. Interpretations were considered correct if 2 or more reviewers agreed on the results.

NCCT/CTA and CTP findings were subsequently compared with DWI. Using DWI as the "criterion standard," findings were denoted as true-positive results if: 1) the abnormalities on NCCT/ CTA corresponded with the same areas as the infarcts on DWI or 2) regional abnormalities on CTP included the area of lacunar infarction on DWI. Abnormalities on NCCT/CTA and CTP were marked as false-positives if: 1) no infarct was present on DWI or 2) the infarct on DWI was in a different location than that of the CTP. A single neuroradiologist graded the amount of chronic small vessel ischemic disease based on a grading scale: 1 (noneminimal), 2 (mild-moderate), 3 (moderate), 4 (moderate-severe), and 5 (severe).

\section{Statistical Analysis}

Intra- and interobserver agreement was calculated by using the Cohen $\kappa$. The sensitivity and specificity of the NCCT/CTA and independent CTP parameters (CBF, CBV, MTT, and TTP) in each area of the brain (basal ganglia and/or thalamus, CSWM, PVWM) were calculated. A $P$ value of $<.05$ was considered statistically significant. Statistical analysis was performed by using

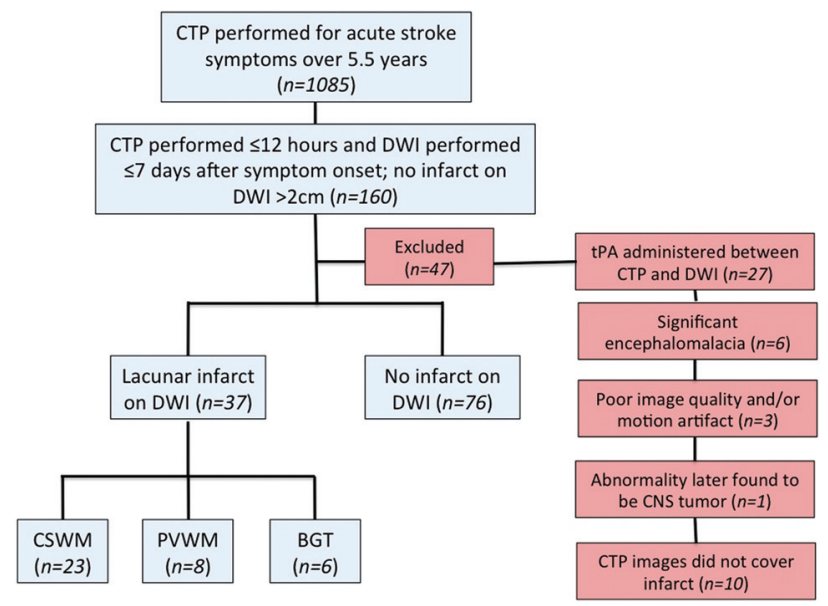

FIG 1. Flowchart of patient population. Infarcts in the cerebellum and brain stem were excluded because these were not adequately covered by the CTP images. BGT indicates basal ganglia and/or thalamus.

Sensitivity, specificity, PPV, and NPV for NCCT/CTA and CTP (based on perfusion parameters) in the detection of acute lacunar infarct

\begin{tabular}{lcccc}
\multicolumn{1}{c}{ Imaging } & & & & \\
\multicolumn{1}{c}{ Modality } & Sensitivity & Specificity & PPV & NPV \\
\hline NCCT $/ C T A$ & $2.7 \%(P<.0001)$ & $100.0 \%(P<.0001)$ & $100.0 \%(P<.99)$ & $68.1 \%(P<.0001)$ \\
CBF & $24.3 \%(P<.003)$ & $97.4 \%(P<.0001)$ & $81.8 \%(P=.07)$ & $72.8 \%(P<.0001)$ \\
CBV & $18.9 \%(P=.0002)$ & $98.7 \%(P<.0001)$ & $87.5 \%(P=.07)$ & $71.4 \%(P<.0001)$ \\
TTP & $48.7 \%(P<.99)$ & $97.4 \%(P<.0001)$ & $90.0 \%(P=.0004)$ & $80.0 \%(P<.0001)$ \\
MTT & $35.1 \%(P=.10)$ & $99.7 \%(P<.0001)$ & $92.9 \%(P=.002)$ & $76.0 \%(P<.0001)$ \\
\hline
\end{tabular}

Note:-NPV indicates negative predictive value; PPV, positive predictive value.
Statistical Analysis Software (SAS; SAS Institute, Cary, North Carolina). Charts were created in Microsoft PowerPoint Version 14.6 (Microsoft, Redmond, Washington).

\section{RESULTS}

\section{Patient Population}

One hundred sixty patients underwent CTP imaging within 12 hours and DWI within 7 days of symptom onset, with or without lacunar infarction on DWI. Of these, 27 were excluded because they received tPA between the CTP and DWI, 10 were excluded because the CTP images did not adequately involve the area of infarction ( 9 in the brain stem or cerebellum and 1 in the PVWM), 6 were excluded because of the presence of encephalomalacia (eg, from prior stroke), 3 were excluded because the examination was limited by motion and/or other types of artifact distortion, and 1 was excluded because the abnormality was later found to be a tumor (Fig 1). Thus, 113 patients were included for this study cohort; 37 (32.7\%) had infarction on DWI, and 76 $(67.3 \%)$ had no infarction. The average age at onset of symptoms was $59.8 \pm 16.9$ years. Sixty (53.1\%) of the included patients were women.

\section{Infarct Timing and Locations}

The average time between symptom onset to CTP imaging was $2.4 \pm 1.1$ hours for all patients; the average time between CTP and MR imaging was $0.9 \pm 1.0$ days. The average degree/grade of chronic small vessel ischemic disease on NCCT was $2.1 \pm 1.3$ for all patients, corresponding to approximately mild-to-moderate disease based on the grading scale used. Of the infarcts noted on DWI, 23 of 37 (62.2\%) were located in the CSWM, 8 of 37 (21.6\%) in the PVWM, and 6 of $37(16.2 \%)$ in the basal ganglia and/or thalamus. The mean NIHSS score for all studied patients was $4.0 \pm 5.0(3.9 \pm 5.6$ for patients with infarct and $4.1 \pm 4.7$ for patients without infarct). Four patients received tPA before both CTP and MR imaging.

\section{Imaging Results}

Regarding lacunar infarcts in all locations within the brain, CTP sensitivity for the detection of lacunae ranged from $18.9 \%$ (CBV) to $48.7 \%$ (TTP) depending on perfusion parameter. The sensitivity of NCCT/CTA was $2.7 \%$. False-positive CTP interpretations, in which the consensus CTP was noted to be positive in a patient without infarct on DWI, were observed in all CTP sequences; no false-positive interpretation occurred in NCCT/CTA. Specificity of CTP ranged from $97.4 \%$ (CBF and TTP) to $98.7 \%$ (CBV and MTT); NCCT/CTA specificity was $100 \%$ (Table). Lacunar infarcts typically appeared on CTP images as regional abnormalities larger than the infarcts observed on DWI (Fig 2).

In all locations, the positive predictive value for CTP ranged from $81.8 \%$ (CBF) to $92.9 \%$ (MTT). The negative predictive value ranged from $71.4 \%$ (CBV) to $80.0 \%$ (TTP). Regarding interpretation of CTP images, interobserver $\kappa$ ranged from 0.38 for $\mathrm{CBF}$ to 0.66 for TTP $(P<.0001$ for all $)$. Interobserver $\kappa$ was 0.88 for DWI $(P<.0001)$ and 0.05 for NCCT/CTA $(P=.18)$. 


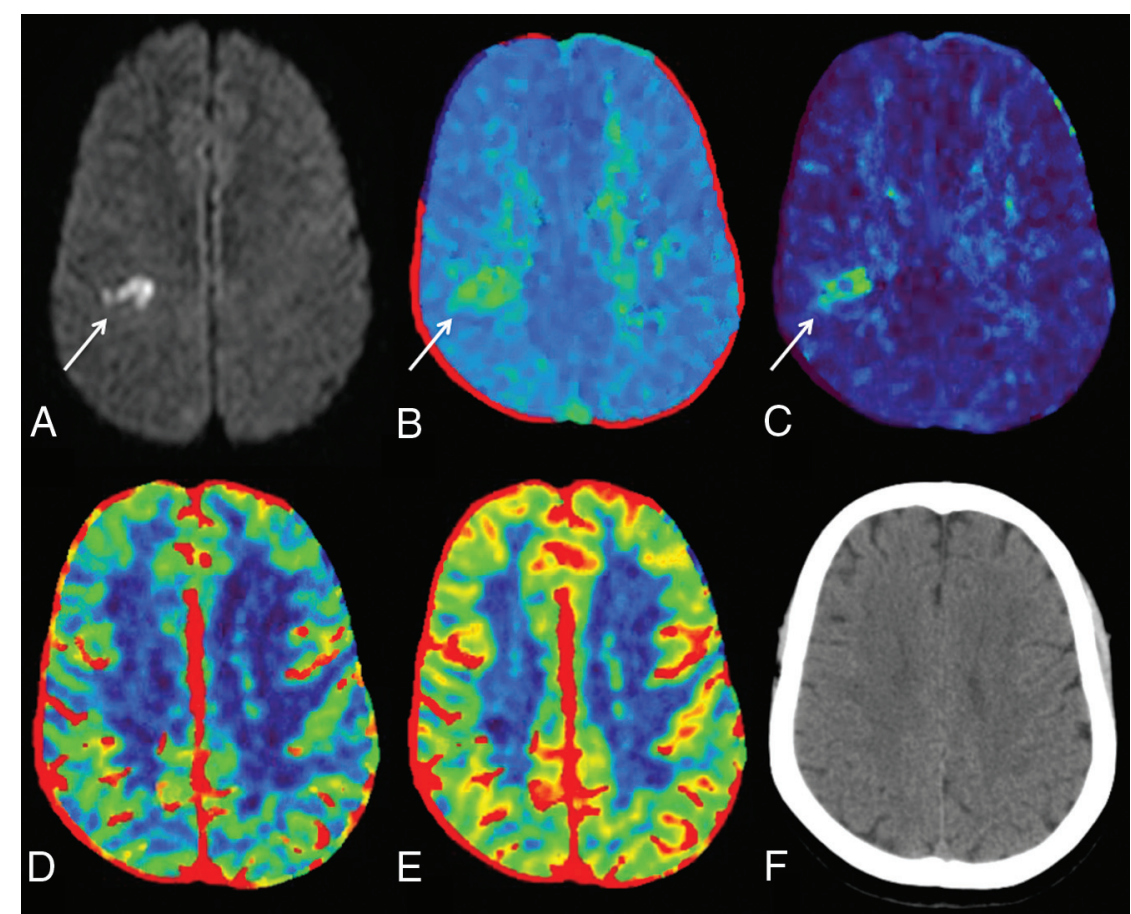

FIG 2. A 49-year-old woman who presented with left-sided facial weakness and dysarthria. Infarct within the right centrum semiovale on DWI (A, white arrow) corresponded with focal abnormalities on TTP $(B)$ and MTT $(C)$ images (white arrow on each); the abnormalities on TTP and MTT were slightly larger than the confirmed infarct on DWI. No abnormalities were identified on CBV $(D)$, CBF $(E)$, or NCCT (F).

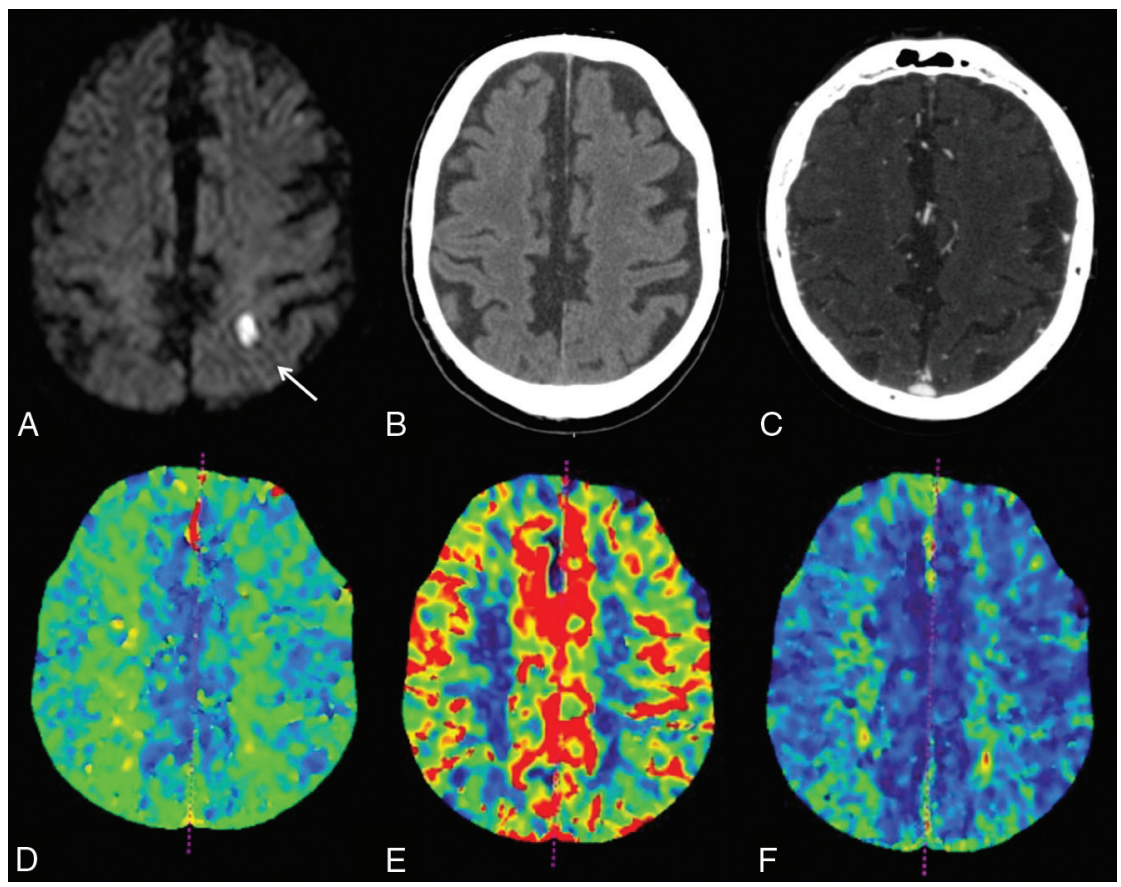

FIG 3. Example of a lacunar infarct not visualized on CTP images. A 74-year-old man who presented with right arm weakness. DWI confirmed a lacunar infarct in the left centrum semiovale ( $A$, white arrow). No abnormalities were noted on NCCT (B), CTA $(C)$, TTP $(D)$, CBF (E), or CBV (F).

Conversely, regarding the capability of CTP to detect lacunar infarcts in different intracranial regions, the sensitivity of CTP was highest in the CSWM with TTP (65.2\%). Within the PVWM, CTP sensitivity was highest with TTP and MTT (37.5\% for both) and lowest with CBF (12.5\%) (Fig 3). None of the lacunar infarcts in the basal ganglia or thalamus were identified by using CTP (0/6). The sensitivity of NCCT/CTA for lacunar infarcts in the basal ganglia and/or thalamus was $16.7 \%$; no lacunar infarcts were identified on NCCT/CTA in the CSWM or PVWM (0/23 and 0/8, respectively). The intracranial location with the highest positive predictive value was the CSWM with MTT (90.9\%).

\section{DISCUSSION}

This study set out to determine the capability of CTP to identify acute lacunar strokes. The results indicate that CTP has high specificity in the detection of lacunar strokes, though its sensitivity is low among all perfusion parameters. CTP is best able to detect infarcts in the CSWM, followed by the PVWM; lacunae were not accurately identified on CTP in the basal ganglia or thalamus. Overall, CTP outperformed NCCT/ CTA in finding lacunar strokes. The sensitivities of TTP and MTT measured in this study were notably higher than those of CBF and CBV, consistent with past studies that have shown these parameters to be inferior to TTP and MTT as indicators of infarct. ${ }^{18}$

Over half of the lacunae noted on DWI in the present study were located in the subcortical white matter with or without involvement of the overlying cortex; the remaining infarcts were located in the PVWM, basal ganglia, and thalamus. Larger-scale studies have noted that most lacunar strokes occur in either the lentiform nuclei or thalamus. ${ }^{19}$ The small number of lentiform and or thalamic lacunae in this study might be related to a relatively small cohort. Nevertheless, it is notable that none of the infarcts in the basal ganglia or thalamus were accurately identified on CTP. The cerebellum and brain stem were not adequately covered by the CTP images and consequently were not assessed in this study, which is a limitation.

A recent study by Das et $\mathrm{al}^{4}$ indicated that CTP had added value to NCCT alone in the setting of lacunar infarcts. However, reported sensitivities of CTP for detecting strokes $<2$ $\mathrm{cm}$ have been quite variable; Mayer et $\mathrm{al}^{20}$ stated that lacunar strokes were difficult to distinguish from artifact on CTP imaging, and other authors have cited sensitivities as low as $0 \%$ and as high as $47.4 \%-62.5 \% .{ }^{10,15,21}$ Furthermore, a systematic review by 
Biesbroek et $\mathrm{al}^{22}$ noted that approximately two-thirds of falsenegative findings on CTP were lacunae, and a study by Eckert et $\mathrm{al}^{23}$ noted that the most common cause of false-negative multimodal CT imaging was lacunar infarcts. Although the reason for this inconsistency among studies is not clear, it is likely that the variability is at least in part because of differences in the designs of the studies (eg, whether there was a known lacunar stroke and whether the reviewers were blinded). In addition, the parameters used in each study, such as time-to-maximum versus TTP, might have contributed to differences in reported sensitivities. The present study provided reviewers with patient NIHSS scores as well as presenting symptoms; thus, the results of this study might better reflect the sensitivity of lacunar infarct detection in a clinical setting.

In addition, differences in reported sensitivities of lacunar infarct detection might be attributed to the use of delay-sensitive singular value decomposition algorithms in some studies. Prior studies by Sasaki et $\mathrm{al}^{24}$ and Kudo et $\mathrm{al}^{25}$ have demonstrated the superior accuracy of delay-corrected singular value decomposition algorithms in CTP in acute infarcts. Delay-sensitive singular value decomposition algorithms have been shown to overestimate MTT prolongation and CBF reduction in acute stroke, thus falsely increasing their sensitivity to infarcts. ${ }^{24}$ A recent study by Cao et $\mathrm{al}^{26}$ also used delay-corrected singular value decomposition to assess for lacunar infarcts; the reviewers were similarly blinded to DWI results, but were aware of the patients' presenting symptoms. The authors of that study reported a superior sensitivity of MTT (56\%) compared with the results of the current study, but similarly noted high specificities $(100 \%$ for CBV, CBF, and MTT).

Although the presence of stroke on CTP appears to be fairly specific for infarction, the infarct size on CTP often did not correlate with the focus of restricted diffusion on DWI. In many patients, the defects on CTP appeared as regional abnormalities corresponding to, but larger than, the location of the lacunar stroke. In contrast, a recent study by Rudilosso et $\mathrm{al}^{15}$ noted focal defects on CTP that more accurately estimated infarct size on DWI. The present study did not set specific CTP thresholds, which might have contributed to the mismatch in infarct sizes observed in CTP and DWI (ie, the area of restricted diffusion might have represented the infarct "core," whereas the observed abnormalities on CTP might have represented a wider zone of ischemia). Alternatively, abnormalities on CTP might have represented the cumulative effect of multiple lacunar infarcts within the same region; although patients with coexistent infarcts $>2 \mathrm{~cm}$ were excluded, some patients had multiple lacunae that might have contributed to additional findings.

This study has limitations shared by any retrospective study. In addition, CTP sections did not adequately cover the brain stem or cerebellum, limiting our evaluation of its diagnostic capabilities in those regions. In addition, there was a temporal difference between the acquisition of CTP and DWI in some patients, which might have biased the results. Also, infarcts might have formed and/or increased in size between the acquisition of CTP and DWI. We sought to minimize any artifact differences by excluding patients who received tPA therapy between the 2 examinations. Next, as stated above, thresholds for CTP were not established for this study. However, the purpose of this study was to assess CTP's ability to identify infarcts rather than measure CTP's capability to estimate infarct core. In addition, only a small number of subjects $(n=6)$ had infarcts within the basal ganglia and/or thalamus, limiting the assessment of CTP capabilities within those regions. Finally, although this study assessed the use of CTP to visually detect positive lacunae based on decreased perfusion and increased delay, no specific measurement or threshold was used.

\section{CONCLUSIONS}

This study found that CTP is superior to NCCT/CTA in the detection of acute lacunar infarcts. The specificity was high for lacunar infarct detection by using all of the studied perfusion parameters, but the sensitivity was lower than DWI. CTP is best able to delineate lacunae within the CSWM, followed by the PVWM, but did not accurately identify lacunar strokes within the basal ganglia or thalamus. Further work is necessary to determine which parameter (eg, TTP, MTT, time-to-maximum) best detects lacunae, and a prospective head-to-head comparison of such parameters might be useful.

Disclosures: Alexander McKinney_RELATED: Provision of Writing Assistance, Medicines, Equipment, or Administrative Support: Vital Images, Comments: provided equipment in 2013 that was used in this study; UNRELATED: Consultancy: Vital Images (a division of Toshiba Medical, Minnetonka, Minnesota), Comments: $<\$ 5000$ total in 2015-16; Expert Testimony: $<\$ 10,000$ legal consultancy in 2015-16. Pascal Salazar-Employment: Vital Images.

\section{REFERENCES}

1. Micheli S, Corea F. Lacunar versus non-lacunar syndromes. Front Neurol Neurosci 2012;30:94-98 CrossRef Medline

2. Altmann M, Thommessen B, Rønning OM, et al. Diagnostic accuracy and risk factors of the different lacunar syndromes. J Stroke Cerebrovasc Dis 2014;23:2085-90 CrossRef Medline

3. Hommel M, Besson G, Le Bas JF, et al. Prospective study of lacunar infarction using magnetic resonance imaging. Stroke 1990;21: 546-54 CrossRef Medline

4. Das T, Settecase F, Boulos M, et al. Multimodal CT provides improved performance for lacunar infarct detection. AJNR Am J Neuroradiol 2015;36:1069-75 CrossRef Medline

5. Caso V, Budak K, Georgiadis D, et al. Clinical significance of detection of multiple acute brain infarcts on diffusion weighted magnetic resonance imaging. J Neurol Neurosurg Psychiatry 2005;76: 514-18 CrossRef Medline

6. Norrving B. Long-term prognosis after lacunar infarction. Lancet Neurol 2003;2:238-45 CrossRef Medline

7. Norrving B. Evolving concept of small vessel disease through advanced brain imaging. J Stroke 2015;17:94-100 CrossRef Medline

8. Arboix A, Martí-Vilalta JL. Lacunar stroke. Expert Rev Neurother 2009;9:179-96 CrossRef Medline

9. Pantoni L, Fierini F, Poggesi A. Thrombolysis in acute stroke patients with cerebral small vessel disease. Cerebrovasc Dis 2014;37: 5-13 CrossRef Medline

10. Hana T, Iwama J, Yokosako S, et al. Sensitivity of CT perfusion for the diagnosis of cerebral infarction. J Med Invest 2014;61:41-45 CrossRef Medline

11. Chamorro A, Sacco RL, Mohr JP, et al. Clinical-computed tomographic correlations of lacunar infarction in the Stroke Data Bank. Stroke 1991;22:175-81 CrossRef Medline

12. Smajlović D, Sinanović O. Sensitivity of the neuroimaging techniques in ischemic stroke. Med Arh 2004;58:282-84 Medline

13. Lai PH, Li JY, Chang CY, et al. Sensitivity of diffusion-weighted magnetic resonance imaging in the diagnosis of acute lacunar infarcts. J Formos Med Assoc 2001;100:370-76 Medline 
14. Wintermark M, Bogousslavsky J. Imaging of acute ischemic brain injury: the return of computed tomography. Curr Opin Neurol 2003; 16:59-63 CrossRef Medline

15. Rudilosso S, Urra X, San Román L, et al. Perfusion deficits and mismatch in patients with acute lacunar infarcts studied with wholebrain CT perfusion. AJNR Am J Neuroradiol 2015;36:1407-12 CrossRef Medline

16. Potter GM, Marlborough FJ, Wardlaw JM. Wide variation in definition, detection, and description of lacunar lesions on imaging. Stroke 2011;42:359-66 CrossRef Medline

17. Leiva-Salinas C, Provenzale JM, Wintermark M. Responses to the $\mathbf{1 0}$ most frequently asked questions about perfusion CT. AJR Am J Roentgenol 2011;196:53-60 CrossRef Medline

18. Tomandl BF, Klotz E, Handschu R, et al. Comprehensive imaging of ischemic stroke with multisection CT. Radiographics 2003;23: 565-92 CrossRef Medline

19. Longstreth WT Jr, Bernick C, Manolio TA, et al. Lacunar infarcts defined by magnetic resonance imaging of 3660 elderly people: the Cardiovascular Health Study. Arch Neurol 1998;55:1217-25 CrossRef Medline

20. Mayer TE, Hamann GF, Baranczyk J, et al. Dynamic CT perfusion imaging of acute stroke. AJNR Am J Neuroradiol 2000;21:1441-49 Medline

21. Maruya J, Yamamoto K, Ozawa T, et al. Simultaneous multi-section perfusion CT and CT angiography for the assessment of acute ischemic stroke. Acta Neurochir (Wien) 2005;147:383-91; discussion 391-92 CrossRef Medline

22. Biesbroek JM, Niesten JM, Dankbaar JW, et al. Diagnostic accuracy of CT perfusion imaging for detecting acute ischemic stroke: a systematic review and meta-analysis. Cerebrovasc Dis 2013;35:493-501 CrossRef Medline

23. Eckert B, Küsel T, Leppien A, et al. Clinical outcome and imaging follow-up in acute stroke patients with normal perfusion CT and normal CT angiography. Neuroradiology 2011;53:79-88 CrossRef Medline

24. Sasaki M, Kudo K, Ogasawara K, et al. Tracer delay-insensitive algorithm can improve reliability of CT perfusion imaging for cerebrovascular steno-occlusive disease: comparison with quantitative single-photon emission CT. AJNR Am J Neuroradiol 2009;30:188-93 CrossRef Medline

25. Kudo K, Sasaki M, Ogasawara K, et al. Difference in tracer delayinduced effect among deconvolution algorithms in CT perfusion analysis: quantitative evaluation with digital phantoms. Radiology 2009;251:241-49 CrossRef Medline

26. Cao W, Yassi N, Sharma G, et al. Diagnosing acute lacunar infarction using CT perfusion. J Clin Neurosci 2016;29:70-72 CrossRef Medline 